\title{
Decreased expression of farnesoid $X$ receptor may indicate poor prognosis in patients with colorectal cancer
}

\author{
Danying Zhang", Shuqiang Weng", Can Cui, Ling Dong, Xizhong Shen \\ Department of Gastroenterology, Zhongshan Hospital, Fudan University, Shanghai, China \\ Contributions: (I) Conception and design: D Zhang, L Dong, S Weng; (II) Administrative support: X Shen; (III) Provision of study materials or \\ patients: L Dong, X Shen; (IV) Collection and assembly of data: D Zhang, C Cui; (V) Data analysis and interpretation: D Zhang, S Weng; (VI) \\ Manuscript writing: All authors; (VII) Final approval of manuscript: All authors. \\ \#These authors contributed equally to this work and should be regarded as co-first authors. \\ Correspondence to: Ling Dong. Department of gastroenterology, Zhongshan Hospital, Fudan University, 180 Fenglin Road, Shanghai, China. \\ Email: dong.ling@zs-hospital.sh.cn.
}

Background: Colorectal cancer (CRC) is one of the most common malignancies worldwide. Many studies showed that increased secretion of bile acid may contribute to the pathogenesis of CRC. Farnesoid $\mathrm{X}$ receptor (FXR) plays an important role in several physiological functions of bile acid metabolism and circulation, while the diagnostic and prognostic values of FXR remain unknown.

Methods: We used immunohistochemistry (IHC) to examine FXR expression in 132 formalin-fixed paraffin-embedded (FFPE) CRC samples, and reverse transcription-quantitative polymerase chain reaction (RT-qPCR) was also conducted to compare the mRNA expression of FXR between 50 fresh CRC tissues and 50 adjacent normal tissues. Immunohistochemical method has been used to compare the protein expression of FXR in FFPE samples, and the associate between FXR expression and clinical characteristics of the patients were also analyzed. Moreover, RT-qPCR has been applied to quantify the mRNA expression of FXR, and the diagnostic value and prognostic value of FXR were analyzed by receiver operating characteristics (ROC) analysis and Kaplan-Meier survival analysis, respectively.

Results: The expression of FXR was significantly decreased in CRC tumor samples compared with normal tissues on both protein and mRNA levels, and the expression of FXR was positively correlated with the tumor size, lymph node metathesis and distant metathesis of the patients. Moreover, the area under curve (AUC) of FXR for the diagnosis of CRC was 0.8088, and low expression of FXR was associated with low overall survival (OS) and disease-free survival (DFS) rate.

Conclusions: FXR was down-regulated in CRC and may serve as potential diagnostic and prognostic biomarkers.

Keywords: Farnesoid X receptor (FXR); bile acid; colorectal cancer (CRC); prognosis; biomarkers

Submitted Dec 06, 2019. Accepted for publication Jun 05, 2020.

doi: $10.21037 /$ tcr-19-2723

View this article at: http://dx.doi.org/10.21037/tcr-19-2723

\section{Introduction}

Colorectal cancer (CRC) is one of the most common malignancies and causes of cancer-related deaths worldwide $(1,2)$, and the incidence of CRC has increased in recent years (3). The pathogenesis of CRC is still unclear, and the prognosis of CRC remains poor $(4,5)$. Therefore, it is of great importance to identify new therapeutic targets for the early diagnosis and prognosis of the disease.

Results from previous studies have shown that increased secretion of bile acid may contribute to the development of CRC (6,7). It has been proposed that bile acid may increase the production of reactive oxygen species and reactive nitrogen species in colonic cells, which damages DNA and 
causes gene mutations and eventually lead to the incidence of the malignancies $(7,8)$. Farnesoid $\mathrm{X}$ receptor (FXR) was discovered by Forman et al. in 1995 and it is a member of the nuclear receptor superfamily (9). FXR plays an important role in several physiological functions, including the metabolism and circulation of bile acid (10). Previous studies suggested that FXR was down-regulated in CRC and may function as a tumor suppressor (11); however, reports on the roles of FXR in CRC are limited, and the clinical value of FXR as diagnostic and prognostic maker in CRC remain unclear. Therefore, the present study evaluated FXR expression patterns in normal colorectal and CRC tissues, and determined its clinical potential. We present the following article in accordance with the REMARK reporting checklist (12) (available at http://dx.doi.org/10.21037/tcr-19-2723).

\section{Methods}

\section{Study populations}

We collected the formalin-fixed paraffin-embedded (FFPE) samples from 132 CRC patients who underwent surgical treatment in the Department of Surgery, Zhongshan Hospital from Jan 2008 to Aug 2008. The patients were followed up for a time interval of 18 up to 110 months with a median follow-up time of $67.6 \pm 37.2$ months. In validation study, 50 fresh CRC tumor samples and the paired adjacent normal tissue were collected (stored in liquid nitrogen) from patients underwent surgical treatment in the Department of Surgery, Zhongshan Hospital from Jan 2017 to Mar 2018. Eligible patients had to meet all of the following criteria: pathologically confirmed primary CRC, no history of malignancy, did not receive any anticancer therapy before surgical treatment. Patients who received radiation or chemotherapy or neoadjuvant preoperatively treatment were excluded. Tumor staging of the patients was done in accordance with the Primary Tumor, Lymph Node, and Metastasis (TNM) classification of the American Joint Commission of Cancer (AJCC) and the Union for International Cancer Control (UICC), seventh edition.

\section{Ethical statement}

The present study was performed with the guidance of the Declaration of Helsinki (as revised in 2013), and it has been approved by the ethical committee of Zhongshan Hospital, Fudan University (ID: y2017-179). All participators have signed the written informed consent form.

\section{Immunobistochemistry (IHC) and scoring}

A standard IHC protocol was used as previously described (13). Briefly, tissue samples collected from surgery were formalin-fixed, paraffin-embedded, and cut into 5 micron thick slices, dewaxed, and rehydrated, followed by heat-induced antigen retrieval in $0.1 \mathrm{mmol} / \mathrm{L}$ citrate solution (pH 6.0) and microwaving for $15 \mathrm{~min}$. Nonspecific binding was blocked by incubating tissue sections in $5 \%$ serum at room temperature for $30 \mathrm{~min}$, followed by incubation with rabbit anti-FXR antibody (Sigma-Aldrich, St. Louis, MO, USA) overnight at $4{ }^{\circ} \mathrm{C}$. Tissues sections were further incubated with biotin-labeled secondary antibody at room temperature for $30 \mathrm{~min}$, and subsequently labeled with avidin-biotin complex and developed in 3,3'-diaminobenzidine dye. All tissue sections underwent hematoxylin-eosin staining for histopathological analysis.

Immunohistochemical results were evaluated using the IHC scoring system. Staining intensity was graded as 0 , no staining; 1, weak staining; 2 , moderate staining; and 3, strong staining. The percentage of positive staining was measured according to the percentage of positively stained cells among the total number of cells as follows: $1,<10 \%$; $2,10-25 \% ; 3,26-50 \%$; and $4,>50 \%$. The two scores were multiplied in five random microscope fields of view to evaluate each tissue section. Each tissue section was eventually given a mean score.

\section{Reverse transcription-polymerase chain reaction (RT-PCR) analysis}

Total RNAs of the fresh tissue samples were isolated by TRIzol ${ }^{\circledast}$ reagent (Invitrogen/Thermo Fisher Scientific, Waltham, MA, USA) and the content of RNA in each sample was measured by NanoDrop-1000 spectrophotometer (Thermo Fisher Scientific, Inc., Wilmington, DE, USA). Next, the mRNAs were reverse transcribed into cDNA by SuperScript ${ }^{\mathrm{TM}}$ IV First-Strand Synthesis System (Invitrogen), and PCR was performed by the SYBR Green MasterMix (MCE, Shanghai, China). All experiments were strictly followed the procedure of the protocol from the manufacturer. Relative mRNA expression of FXR is quantified to the expression of GAPDH by 2$\Delta \Delta \mathrm{Ct}$ methods. The primers were synthesized by Sangon Biotech (Shanghai, China), and the sequences were as follows: FXR forward, 5'-CCTCATTGTCTCCCC GACTTATCCT-3' and reverse, 3'-GCCTCTA GAAAGCAGTGTTCACTTT-5'; GAPDH forward, 


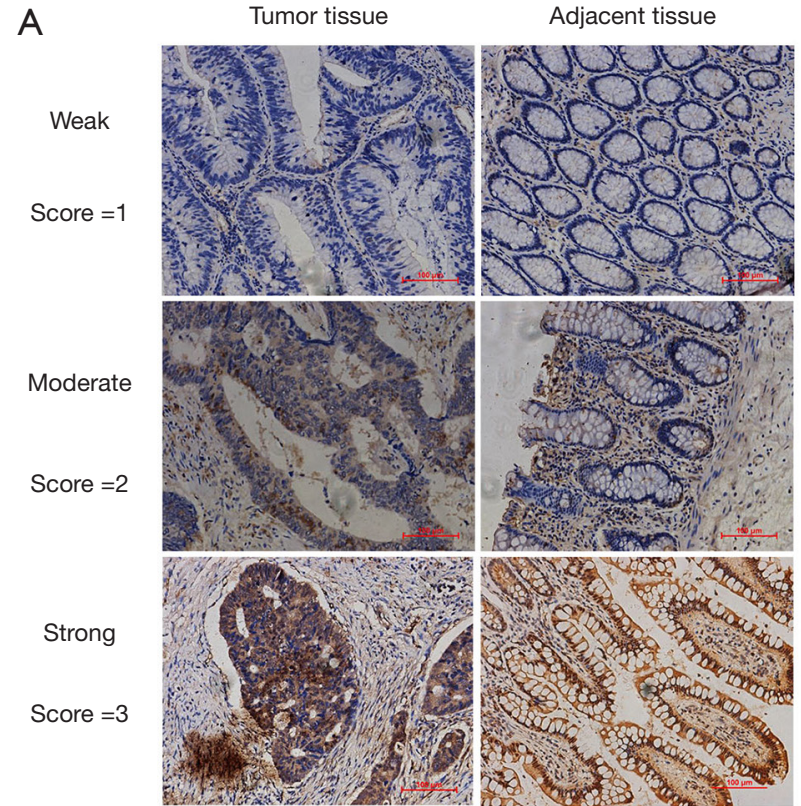

B

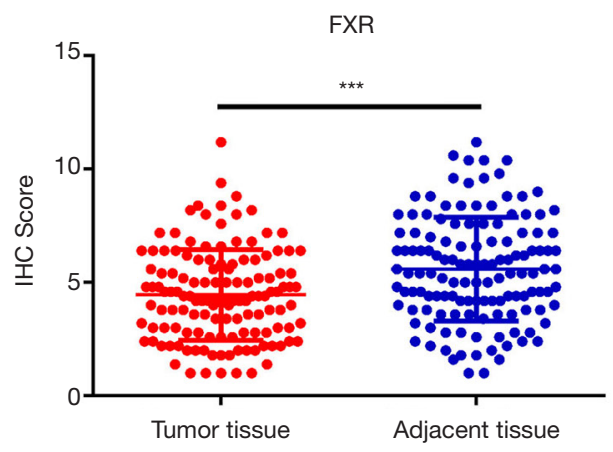

Figure 1 Decreased expression of farnesoid $\mathrm{X}$ receptor in formalin-fixed paraffin-embedded colorectal cancer tissue samples. (A) Representative images of the immunohistochemical staining results. Magnification 200x. (B) Comparison of the score of the immunohistochemical staining for farnesoid $\mathrm{X}$ receptor between colorectal cancer tissues and the adjacent normal tissue. ${ }^{* * *} \mathrm{P}<0.001$.

5'-GCCGCATCTTCTTTTGCGTCGC-3' and reverse, 5'-TCCCGTTCTCAGCCTTGACGGT-3'.

\section{Statistical analysis}

Overall survival (OS) was defined as time between the first day of surgical treatment and date of death from any cause. Disease-free survival (DFS) was defined as the time interval between the date of surgery and the date of detection of recurrence or the date of last follow-up without recurrence for CRC. To investigate the independent prognostic value of FXR and the clinicopathological variables, multivariate Cox regression analyses of OS and DFS were performed. GraphPad prism 7.0 (GraphPad Software, San Diego, CA, USA) was used for the statistical analyses. Paired student $t$-test was used to compare the difference between tumors and normal tissues. Chi-squared test was used for the analysis between FXR expression and the clinical characteristics of the patients. Kaplan-Meier methods were used for the survival analysis. $\mathrm{P}<0.05$ was considered statistically significant.

\section{Results}

\section{Decreased expression of FXR in FFPE CRC tissue samples}

Tumor samples from 141 patients were sent to the clinical laboratory for FXR assays by IHC, and evaluable assay results were obtained for 132 patients. Among the 9 patients with unevaluable results, 5 had insufficient tumor cells, 2 had extensive necrosis, and 2 had poor tumor preservation. Then, we performed immunohistochemical staining to compare the expression of FXR between CRC tissues and the adjacent normal tissue. The representative images of the FXR staining results were shown in Figure $1 \mathrm{~A}$. We found that the average immunohistochemical score of FXR for tumor tissue vs. adjacent tissue was $4.45 \pm 2.01$ vs. $5.58 \pm 2.29$ (Figure $1 B, \mathrm{P}<0.001$ ).

\section{Association between the expression of FXR and clinical characteristics of the patients}

Then, we divided the 132 patients into FXR low and FXR high groups based on their immunohistochemical score of $\leq 4$ and $>4$, and the association between the expression of FXR and clinical characteristics of the patients were analyzed. The results were shown in Table 1. Among the 132 patients, low expression of FXR was associated with the tumor size $(\mathrm{P}=0.008)$, lymph node metastasis $(\mathrm{P}=0.008)$ and distant metastasis $(\mathrm{P}=0.027)$ of the patients; while no association was detected between FXR expression and age $(\mathrm{P}=0.375)$, gender $(\mathrm{P}=0.640)$, degree of tumor differentiation $(\mathrm{P}=0.590)$ and TNM stage $(\mathrm{P}=0.207)$.

\section{FXR was decreased in fresh CRC tissue samples and may serve as potential diagnostic biomarkers}

Next, to further explore the role of FXR in CRC, 50 fresh CRC tumor tissue samples and the paired-adjacent 
Table 1 Association between farnesoid $\mathrm{X}$ receptor expression and characteristics of the patients with colorectal cancer

\begin{tabular}{|c|c|c|c|}
\hline \multirow{2}{*}{ Clinical characteristics } & \multicolumn{2}{|c|}{ Farnesoid $\mathrm{X}$ receptor } & \multirow{2}{*}{$P$ value } \\
\hline & Low $(n=53)$ & High $(n=79)$ & \\
\hline Age (years) & & & 0.375 \\
\hline$\geq 60(n=66)$ & 29 & 37 & \\
\hline$<60(n=66)$ & 24 & 42 & \\
\hline Gender & & & 0.640 \\
\hline Male $(n=73)$ & 28 & 45 & \\
\hline Female $(n=59)$ & 25 & 34 & \\
\hline Tumor size $(\mathrm{cm})$ & & & $0.008^{* *}$ \\
\hline$\geq 4(n=78)$ & 24 & 54 & \\
\hline$<4(n=54)$ & 29 & 25 & \\
\hline Tumor differentiation & & & 0.590 \\
\hline Low-medium $(n=101)$ & 43 & 58 & \\
\hline Medium-high ( $\mathrm{n}=22)$ & 7 & 15 & \\
\hline Unknown (n=9) & 3 & 6 & \\
\hline TNM stage & & & 0.207 \\
\hline$I-I I(n=56)$ & 26 & 30 & \\
\hline III-IV (n=76) & 27 & 49 & \\
\hline $0(n=74)$ & 21 & 53 & \\
\hline Lymph node metastasis & & & $0.008^{\star *}$ \\
\hline $1-3(n=41)$ & 23 & 18 & \\
\hline$\geq 4(n=17)$ & 9 & 8 & \\
\hline Distant metastasis & & & $0.027^{*}$ \\
\hline No $(n=113)$ & 41 & 72 & \\
\hline Yes $(n=19)$ & 12 & 7 & \\
\hline
\end{tabular}

${ }^{*} \mathrm{P}<0.05,{ }^{* *} \mathrm{P}<0.01$.

normal tissues were collected. RT-qPCR was performed to analysis the expression of FXR. As shown in Figure 2A, the expression of FXR was markedly decreased in CRC tumor samples compared with the adjacent tissues $(\mathrm{P}<0.001)$. Moreover, we also performed receiver operating characteristics (ROC) analysis to investigate the potential diagnostic value of FXR for CRC. The results showed that the area under curve (AUC) of FXR for the diagnosis of CRC was 0.8088 (Figure 2B, 95\% confidence interval 0.7203 to 0.8973$)$. These results suggested that FXR was a sensitive biomarker for the diagnosis of CRC.
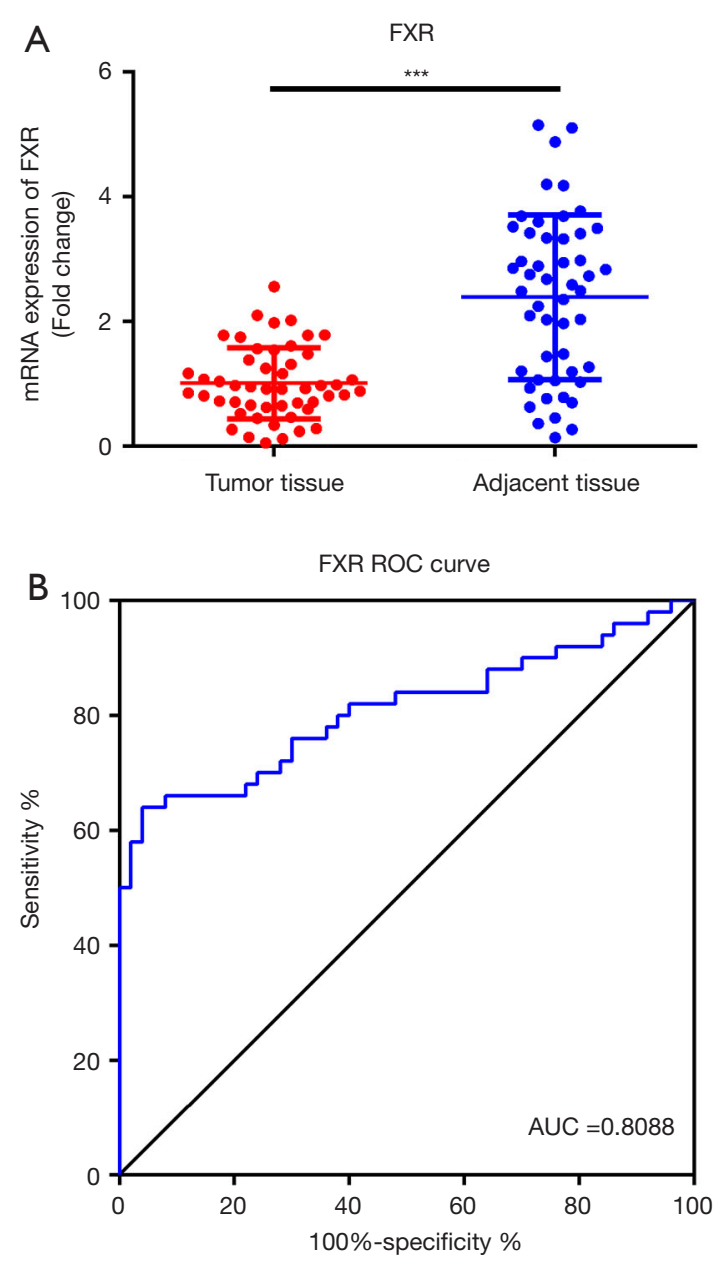

Figure 2 Farnesoid $\mathrm{X}$ receptor may function as a diagnostic biomarker in colorectal cancer. (A) Comparison of the mRNA expression of farnesoid $\mathrm{X}$ receptor between colorectal cancer tissues and the adjacent normal tissue by RT-qPCR methods. (B) Results of receiver operating characteristics analysis for evaluating the diagnostic value of farnesoid $\mathrm{X}$ receptor for colorectal cancer. ${ }^{* * *} \mathrm{P}<0.001$. RT-qPCR, reverse transcription-quantitative polymerase chain reaction.

\section{Decreased expression of FXR may indicate poor prognosis of the patients}

During follow-up, 79 of the 132 CRC patients survived; of the 53 died patients, 50 died from CRC recurrence or metastasis, 1 died from a car accident, 1 died from heart disease, 1 died from cerebrovascular disease and these 3 the patients were excluded for the survival analysis. KaplanMeier survival analysis was performed to evaluate the correlation between FXR expression and the OS as well as 

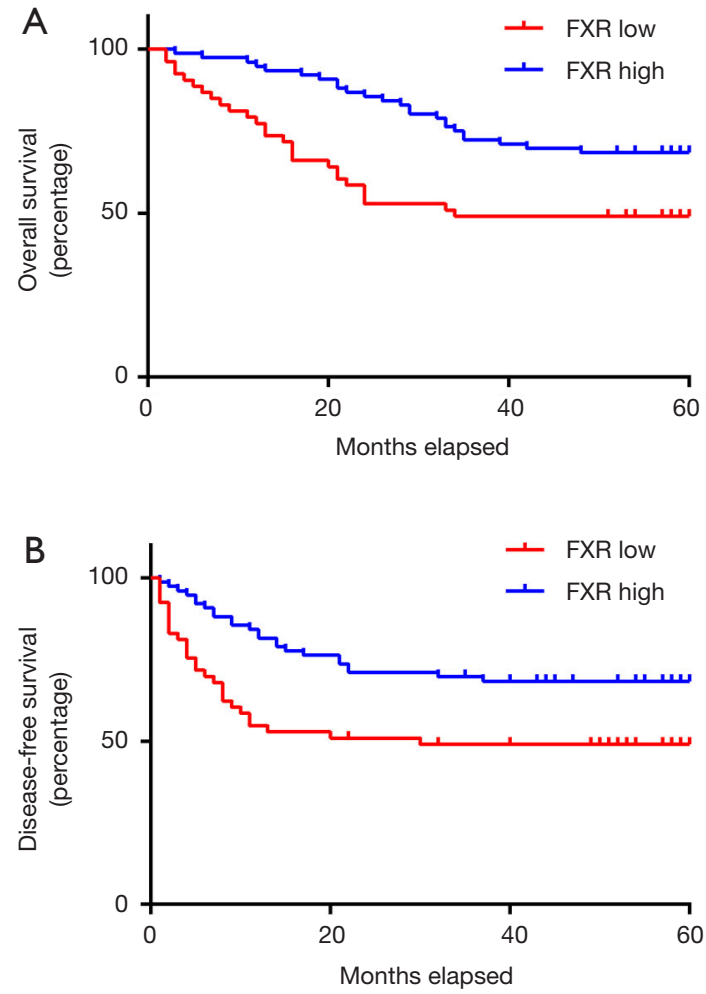

Figure 3 Farnesoid $\mathrm{X}$ receptor may function as a prognostic biomarker in colorectal cancer. Kaplan-Meier survival analysis was performed to evaluate the correlation between farnesoid $\mathrm{X}$ receptor expression and the overall survival $(\mathrm{P}=0.006)(\mathrm{A})$ and disease-free survival $(\mathrm{P}=0.007)(\mathrm{B})$ of the patients. the DFS of patients. We found that OS (Figure $3 A, \mathrm{P}=0.006$ ) and DFS rate (Figure $3 B, \mathrm{P}=0.007$ ) were significantly higher in FXR high group compared with the FXR low group, indicating that decreased expression of FXR may predict poor prognosis of the CRC patients. In multivariate analysis, tumor size, TNM stage, lymph node metastasis, distant metastasis and FXR expression were identified as independent prognostic factors of overall patients' survival (Table 2). Tumor size, TNM stage, lymph node metastasis and FXR expression were also identified as independent prognostic factors of disease-free patients' survival (Table 2). When all standard prognostic clinical variables were included as co-variables in a Cox proportional hazards model, there was still evidence that FXR expression was significantly associated with OS (HR $=0.325,95 \%$ CI: $0.074-0.715, \mathrm{P}=0.0045)$ and $\mathrm{DFS}(\mathrm{HR}=0.384,95 \% \mathrm{CI}$ : 0.069-0.812, $\mathrm{P}=0.0071)$. Estimated effects were similar in the model without stratification and sensitivity analysis further confirmed FXR expression was still the strongest prognostic factor.

\section{Discussion}

In this study, the potential diagnostic and prognostic values of FXR in CRC were evaluated. We found that FXR was decreased in CRC tissue samples on both protein and mRNA levels, and FXR expression was negatively associated

Table 2 Prognostic values of several factors in a multivariable analysis of OS and DFS for 132 patients with colorectal cancer

\begin{tabular}{lcccc}
\hline \multirow{2}{*}{ Clinicopathological variables } & \multicolumn{2}{c}{ Overall survival } & \multicolumn{2}{c}{ Disease-free survival } \\
\cline { 2 - 3 } Age $(\geq 60$ vs. $<60)$ & HR $(95 \% \mathrm{Cl})$ & $\mathrm{P}$ value & $\mathrm{HR}(95 \% \mathrm{Cl})$ \\
Sex (female vs. male) & $1.371(1.004-2.357)$ & 0.0526 & $1.254(1.001-2.845)$ & 0.0593 \\
Tumor size (<4 vs. $\geq 4 \mathrm{~cm})$ & $1.032(0.605-1.737)$ & 0.9274 & $1.164(0.662-2.107)$ & 0.5483 \\
Tumor differentiation (low-medium vs. medium-high) & $1.721(1.187-2.964)$ & 0.0153 & $1.458(1.143-3.021)$ & 0.0195 \\
TNM stage (I-II vs. III-IV) & $0.695(0.372-1.417)$ & 0.2987 & $0.612(0.285-1.392)$ & 0.3184 \\
Lymph node metastasis (0 vs. 1-4) & $2.915(1.435-5.827)$ & 0.0037 & $2.029(1.088-3.854)$ & 0.0217 \\
Distant metastasis (no vs. yes) & $3.948(2.785-6.975)$ & 0.0029 & $3.536(2.027-6.219)$ & 0.0016 \\
FXR expression (low vs. high) & $2.536(1.072-3.876)$ & 0.0378 & $1.935(0.783-4.036)$ & 0.1636 \\
\hline
\end{tabular}

OS, overall survival; DFS, disease-free survival; FXR, farnesoid $X$ receptor. 
with the growth and metathesis of the tumor. Thus, decreased expression of FXR may indicate undesired longterm clinical outcomes of CRC patients.

Previous studies have mostly shown a correlation between FXR and hepatobiliary diseases. The decline in FXR expression has been shown to cause a variety of bile acid-related diseases, including intrahepatic cholestasis (14), gallstones (15), and primary biliary cirrhosis (16). FXR deficiency has been reported to be associated with the incidence of hepatocellular carcinoma $(17,18)$. In CRC, it has been reported that FXR may function as a tumor suppressor via inhibiting the oncogenic behavior of the CRC cells (11). However, reports on the function of FXR in CRC was limited, especially its clinical significance remains to be further explored.

In the present study, the expression of FXR in CRC samples were examined. We found that FXR expression in CRC tumor tissues was significantly lower than tumoradjuvant normal tissues on both mRNA and protein levels, which is consistent with its expression pattern in hepatocellular carcinoma and gastric cancer $(18,19)$. Besides, the expression level of FXR was negatively associated with the tumor size and metathesis in CRC patients, which further confirmed its tumor suppressive roles. Taken together, these results suggested that FXR was down-regulated in CRC, and may exert anti-tumor function in the pathogenesis of CRC.

Results of previous works also suggested the diagnostic and prognostic value of FXR in different disease. In fact, FXR may play different roles in different cancers, either as tumor suppressor or oncogene. Recently, You et al. reported that FXR was up-regulated in non-small cell lung cancer, and increased expression of FXR was associated with the poor prognosis of the patients (20). Giaginis et al. suggested that FXR function as a tumor suppressor in pancreatic cancer and elevated expression of FXR can lead to better prognosis of the patient (21). Wang et al. found that FXR was associated with the poor prognosis of gallbladder cancer (22). However, the clinical significance of FXR as potential biomarkers in CRC remains unclear. In the present study, we conducted ROC analysis and found that the AUC of FXR was 0.8088 , which suggested the clinical value of FXR as a sensitive biomarker in CRC diagnosis; moreover, the OS and DFS rate were significantly lower in patients with low FXR expression, suggesting predictive value of FXR in CRC. Taken together, these results confirmed the value of FXR as diagnostic and prognostic marker in CRC, suggesting that monitoring the changes in the expression level of FXR may be beneficial for the diagnosis and treatment of the CRC.

Due to its retrospective nature, some limitations of this study should be addressed. First, because of cost and practical issues, we only included 50 fresh tissue samples for the RTqPCR analysis, and more samples should be analyzed in future to avoid the possible false-positive results caused by small sample size. Second, further investigation should be performed to explore the underlying mechanism and signaling pathway(s) of FXR in CRC as a tumor suppressor.

In conclusion, we found that FXR was down-regulated in CRC patients, and we for the first time analyzed the clinical potential of FXR in early diagnosis, prognosis and treatment of CRC. Our data suggest that FXR expression in CRCs is associated with patients survival and may thus serve as diagnostic and prognostic marker. Larger studies of different ethnic populations, especially with detailed clinical information, are needed to confirm our findings. The results from present study suggested a promising therapeutic target for CRC, and the therapeutic benefits of FXR agonist for $\mathrm{CRC}$ are warranted to investigate in the future.

\section{Acknowledgments}

We would like to thank our anonymous reviewers for their valuable time and helpful comments.

Funding: None.

\section{Footnote}

Reporting Checklist: The authors have completed the REMARK reporting checklist. Available at http://dx.doi. org/10.21037/tcr-19-2723

Data Sharing Statement: Available at http://dx.doi. org/10.21037/tcr-19-2723

Conflicts of Interest: All authors have completed the ICMJE uniform disclosure form (available at http://dx.doi. org/10.21037/tcr-19-2723). The authors have no conflicts of interest to declare.

Ethical Statement: The authors are accountable for all aspects of the work in ensuring that questions related to the accuracy or integrity of any part of the work are appropriately investigated and resolved. The present study was performed with the guidance of the Declaration of Helsinki (as revised in 2013), and it has been approved by the ethical committee of 
Zhongshan Hospital, Fudan University (ID: y2017-179). All participators have signed the written informed consent form.

Open Access Statement: This is an Open Access article distributed in accordance with the Creative Commons Attribution-NonCommercial-NoDerivs 4.0 International License (CC BY-NC-ND 4.0), which permits the noncommercial replication and distribution of the article with the strict proviso that no changes or edits are made and the original work is properly cited (including links to both the formal publication through the relevant DOI and the license). See: https://creativecommons.org/licenses/by-nc-nd/4.0/.

\section{References}

1. Montal ED, Bhalla K, Dewi RE, et al. Inhibition of phosphoenolpyruvate carboxykinase blocks lactate utilization and impairs tumor growth in colorectal cancer. Cancer Metab 2019;7:8.

2. Battaglin F, Puccini A, Ahcene Djaballah S, et al. The impact of panitumumab treatment on survival and quality of life in patients with RAS wild-type metastatic colorectal cancer. Cancer Manag Res 2019;11:5911-24.

3. Winkels RM, van Zutphen M. Multiple outcomes in a meta-analysis of dietary patterns and colorectal cancer. Eur J Clin Nutr 2020:74:208.

4. Zhang J, Liu KC, Peng P, et al. Upregulation of nectin-4 is associated with ITGB1 and vasculogenic mimicry and may serve as a predictor of poor prognosis in colorectal cancer. Oncol Lett 2019:18:1163-70.

5. Feng Z, Shi X, Zhang Q, et al. Analysis of clinicopathological features and prognosis of 1315 cases in colorectal cancer located at different anatomical subsites. Pathol Res Pract 2019;215:152560.

6. Lee S, Lee K, Yoon S, et al. Anomalies in network bridges involved in bile Acid metabolism predict outcomes of colorectal cancer patients. PLoS One 2014;9:e107925.

7. Ridlon JM, Wolf PG, Gaskins HR. Taurocholic Acid Metabolism by Gut Microbes and Colon Cancer. Gut Microbes 2016;7:201-15.

8. Gadaleta RM, Garcia-Irigoyen O, Moschetta A. Bile Acid and Colon Cancer: Is FXR the Solution of the Conundrum. Mol Aspects Med 2017;56:66-74.

9. Forman BM, Goode E, Chen J, et al. Identification of a nuclear receptor that is activated by farnesol metabolites. Cell 1995;81:687-93.

10. Chiang JY, Pathak P, Liu H, et al. Intestinal Farnesoid $\mathrm{X}$ Receptor and Takeda G Protein Couple Receptor 5
Signaling in Metabolic Regulation. Dig Dis 2017;35:241-5.

11. Bailey AM, Zhan L, Maru D, et al. FXR silencing in human colon cancer by DNA methylation and KRAS signaling. Am J Physiol Gastrointest Liver Physiol 2014;306:G48-8.

12. McShane LM, Altman DG, Sauerbrei W, et al. REporting recommendations for tumour MARKer prognostic studies (REMARK). Br J Cancer 2005;93:387-91.

13. Chen XL, Xie KX, Yang ZL, et al. Expression of FXR and HRG and Their Clinicopathological Significance in Benign and Malignant Pancreatic Lesions. Int J Clin Exp Pathol 2019;12:2111-20.

14. Vitale G, Gitto S, Vukotic R, et al. Familial Intrahepatic Cholestasis: New and Wide Perspectives. Dig Liver Dis 2019;51:922-33.

15. Yu DD, Andrali SS, Li HZ, et al. Novel FXR (Farnesoid X Receptor) Modulators: Potential Therapies for Cholesterol Gallstone Disease. Bioorg Med Chem 2016;24:3986-93.

16. Floreani A, Mangini C. Primary Biliary Cholangitis: Old and Novel Therapy. Eur J Intern Med 2018;47:1-5.

17. Guo F, Xu Z, Zhang Y, et al. FXR induces SOCS3 and suppresses hepatocellular carcinoma. Oncotarget 2015;6:34606-16.

18. Kainuma M, Takada I, Makishima M, et al. Farnesoid X Receptor Activation Enhances Transforming Growth Factor $\beta$-Induced Epithelial-Mesenchymal Transition in Hepatocellular Carcinoma Cells. Int J Mol Sci 2018;19:1898.

19. Duan JH, Fang L. MicroRNA-92 promotes gastric cancer cell proliferation and invasion through targeting FXR. Tumour Biol 2014;35:11013-9.

20. You W, Li L, Sun D, et al. Farnesoid X Receptor Constructs an Immunosuppressive Microenvironment and Sensitizes FXR (high) PD-L1 (low) NSCLC to Anti-PD-1 Immunotherapy. Cancer Immunol Res 2019;7:990-1000.

21. Giaginis C, Koutsounas I, Alexandrou P, et al. Elevated Farnesoid X Receptor (FXR) and Retinoid X Receptors (RXRs) expression is associated with less tumor aggressiveness and favourable prognosis in patients with pancreatic adenocarcinoma. Neoplasma 2015;62:332-41.

22. Wang W, Yin X, Li G, et al. Expressions of farnesoid X receptor and myeloid cell leukemia sequence 1 protein are associated with poor prognosis in patients with gallbladder cancer. Chin Med J (Engl) 2014;127:2637-42.

Cite this article as: Zhang D, Weng S, Cui C, Dong L, Shen X. Decreased expression of farnesoid $\mathrm{X}$ receptor may indicate poor prognosis in patients with colorectal cancer. Transl Cancer Res 2020;9(7):4290-4296. doi: 10.21037/tcr-19-2723 